\title{
Helicobacter pylori cagL amino acid polymorphisms and its association with gastroduodenal diseases
}

\author{
Sanket Kumar Shukla $\cdot$ Kashi Nath Prasad • Aparna Tripathi • \\ Virendra Jaiswal · Jahanarah Khatoon - Uday Chand Ghsohal · \\ Narendra Krishnani $\cdot$ Nuzhat Husain
}

Received: 27 April 2012/ Accepted: 2 August 2012/Published online: 1 September 2012

(C) The International Gastric Cancer Association and The Japanese Gastric Cancer Association 2012

\begin{abstract}
CagL is a pilus protein of Helicobacter pylori that interacts with host cellular $\alpha 5 \beta 1$ integrins through its arginine-glycine-aspartate (RGD) motif, guiding proper positioning of the T4SS and translocation of CagA. Deletion or sequence variations of cagL significantly diminished the ability of $H$. pylori to induce secretion of IL- 8 by the host cell. Therefore, this study was undertaken to investigate the association of cagL and its amino acid sequence polymorphisms with gastric cancer (GC), peptic ulcer disease (PUD), and non-ulcer dyspepsia (NUD) as there are no such studies from India. In total, 200 adult patients (NUD 120, PUD 30, GC 50) who underwent an upper gastrointestinal endoscopy were enrolled. $H$. pylori infection was diagnosed by rapid urease test, culture, histopathology, and PCR. The collected isolates were screened for cagL genotype by PCR and assessed for amino acid sequence polymorphisms using
\end{abstract}

Electronic supplementary material The online version of this article (doi:10.1007/s10120-012-0189-7) contains supplementary material, which is available to authorized users.

S. K. Shukla · K. N. Prasad (ه) · A. Tripathi · V. Jaiswal ·

J. Khatoon

Department of Microbiology, Sanjay Gandhi Postgraduate

Institute of Medical Sciences, Lucknow 226 014, India

e-mail:knprasad@sgpgi.ac.in

U. C. Ghsohal

Department of Gastroenterology, Sanjay Gandhi Postgraduate

Institute of Medical Sciences, Lucknow 226 014, India

N. Krishnani

Department of Pathology, Sanjay Gandhi Postgraduate Institute of Medical Sciences, Lucknow 226 014, India

N. Husain

Department of Pathology, Ram Manohar Lohia Institute

of Medial Sciences, Lucknow, India sequence translation. The prevalence of $H$. pylori infection in study population was $52.5 \%$. Most of the isolates were cagL genopositive $(86.6 \%)$, and all had RGD motif in their amino acid sequences. D58 and K59 polymorphisms in cagLgenopositive strains were significantly higher in GC patients $(P<0.05)$. Combined D58K59 polymorphism was associated with higher risk of GC (3.8-fold) when compared to NUD. In conclusion, H. pylori cagL amino acid polymorphisms such as D58K59 are correlated with a higher risk of $\mathrm{GC}$ in the Indian population. Further studies are required to know the exact role of particular cagL amino acid polymorphisms in the pathogenicity of $H$. pylori infection.

Keywords Helicobacter pylori genotypes .

Gastric cancer $\cdot$ CagL polymorphism

$\begin{array}{ll}\text { Abbreviations } \\ \text { GC } & \text { Gastric cancer } \\ \text { PUD } & \text { Peptic ulcer disease } \\ \text { NUD } & \text { Non-ulcer dyspepsia } \\ \text { H. pylori } & \text { Helicobacter pylori } \\ \text { T4SS } & \text { Type 4 secretary system }\end{array}$

\section{Introduction}

The incidence of Helicobacter pylori (H. pylori) infection continues to be higher in developing countries and is estimated at $3-10 \%$ per year in contrast to approximately $1 \%$ per year in developed countries [1]. Many virulence factors have been identified in $H$. pylori: urease, the blood group antigen-binding adhesin ( $b a b A)$, the cag pathogenicity island, the vacuolating cytotoxin (vacA), and H. pylori neutrophil-activating protein (HP-NAP) are the important ones [2]. 
A 40-kb cag pathogenicity island (cag PAI) encodes T4SS, which injects the CagA oncoprotein as well as peptidoglycan into host cells, resulting in induction of potent proinflammatory chemokines such as interleukin (IL)-8 [3]. Translocated CagA undergoes tyrosine phosphorylation by Src, leading to actin cytokine rearrangements, scattering, and elongation of infected host cells.

Previously we reported a high prevalence of $\mathrm{cagA}$ in our study populations [ $80 \%$ in gastric cancer (GC), $83.3 \%$ in peptic ulcer disease (PUD), and $76.7 \%$ in non-ulcer dyspepsia (NUD)] and also found no correlation of cagA with GC and PUD [4]. Therefore, it is likely that $c a g A$ cannot be considered as the sole virulence marker for the determination of disease outcome, at least in India. Hence, it is possible that some other genes of $c a g A$-PAI are responsible for pathogenicity and disease outcome. Genotyping of cag $L$ would be the better molecular tool to understand this problem. CagL is a pilus protein that interacts with host cellular $\alpha 5 \beta 1$ integrins through its arginine-glycine-aspartate (RGD) motif, guiding proper positioning of the T4SS and translocation of CagA [5]. Consequent activation of host signaling pathways stimulates the transcription factor NF- $\kappa \mathrm{B}$ and upregulates inflammation-associated genes such as IL-8.

Therefore, in the current study our aim was to determine the prevalence of $c a g L$ genopositivity and its amino acid polymorphisms in patients with GC, PUD, and NUD.

\section{Materials and methods}

Patient population

We enrolled 200 adult patients (NUD 120, PUD 30, GC 50) undergoing upper gastrointestinal endoscopies at Sanjay Gandhi Postgraduate Institute of Medical Sciences, Lucknow, India, between September 2007 and October 2010. The diagnosis of gastroduodenal diseases was based on clinical, endoscopic, and histopathological examination. Patients with NUD were considered as controls in our study. The ethics committee of the institute granted approval for the study, and all the patients gave their consent to participate. Subjects who had received antimicrobial therapy, $\mathrm{H}_{2}$-receptor blockers, proton-pump inhibitors, and non-steroidal antiinflammatory drugs in the 30 days before the endoscopies were excluded from the study.

Detection of $H$. pylori infection

During each endoscopic examination, antral biopsies were obtained and subjected to the following tests: rapid urease test (RUT), culture, histopathology, and H. pylori-specific
ureA polymerase chain reaction (PCR) following the standard protocol as described elsewhere [6]. H. pylori infection was diagnosed if culture was positive or any two of the remaining three tests were found to be positive.

\section{H. pylori genotyping}

DNA was isolated from the bacterial growth and H. pyloripositive gastric tissues using the QIAamp DNA mini kit (Qiagen, Hilden, Germany) as per the manufacturer's instructions and subjected to PCR for the presence of cagA and cagL genotypes of $H$. pylori, as described previously $[7,8]$.

CagL-genosequencing for translating into amino acid sequences

The DNA fragments amplified by the cagL region were purified by the Qiagen quick PCR purification kit (Qiagen) and sequenced using both the forward and reverse primers to verify that they truly represented the $H$. pylori cytotoxinassociated gene L. Sequencing was performed by Eurofins, MWG operon (Bangalore, India), and analyzed by the BLAST program at the National Center for Biotechnology Information. Furthermore, amino acid sequences of $c a g L$ were predicted by the standard code, and aligned by the Expasy online tool for translation (http://www.expasy.org).

Statistical analysis

Data were analyzed using SPSS software (version 12.0; SPSS, Chicago, IL, USA). H. pylori genotypes and cagL amino acid polymorphism status in relationship to gastroduodenal diseases were assessed by Chi square test. All $P$ values were two sided and considered significant when $<0.05$.

\section{Results}

Patient characteristics

A total of 200 patients (mean age, $46.34 \pm 14.71$ years; 123 males) were enrolled and their distributions were as follows: GC 50 (mean age, $52.12 \pm 12.41$ years; 37 males), PUD 30 (mean age, $47.10 \pm 14.71$ years; 18 males), and NUD 120 (mean age, $43.77 \pm 14.99 ; 68$ males).

Detection of H. pylori infection

The prevalence of $H$. pylori infection in our study population was $52.5 \%$. H. pylori infection was significantly 
Fig. 1 The comparison of $c a g L$ amino acid sequences between J99 Helicobacter pylori strains and nine clinical isolates submitted in the NCBI gene bank. The $\rightarrow$ indicates the RGD motif, and the asterisks indicate sequences with variations at the $35,58,59,60$, 62 , and 122 residues. The nucleotides marked "." indicate identical sequence regions to the J99 sequence, and nucleotides marked "-" are donated as an unamplified region

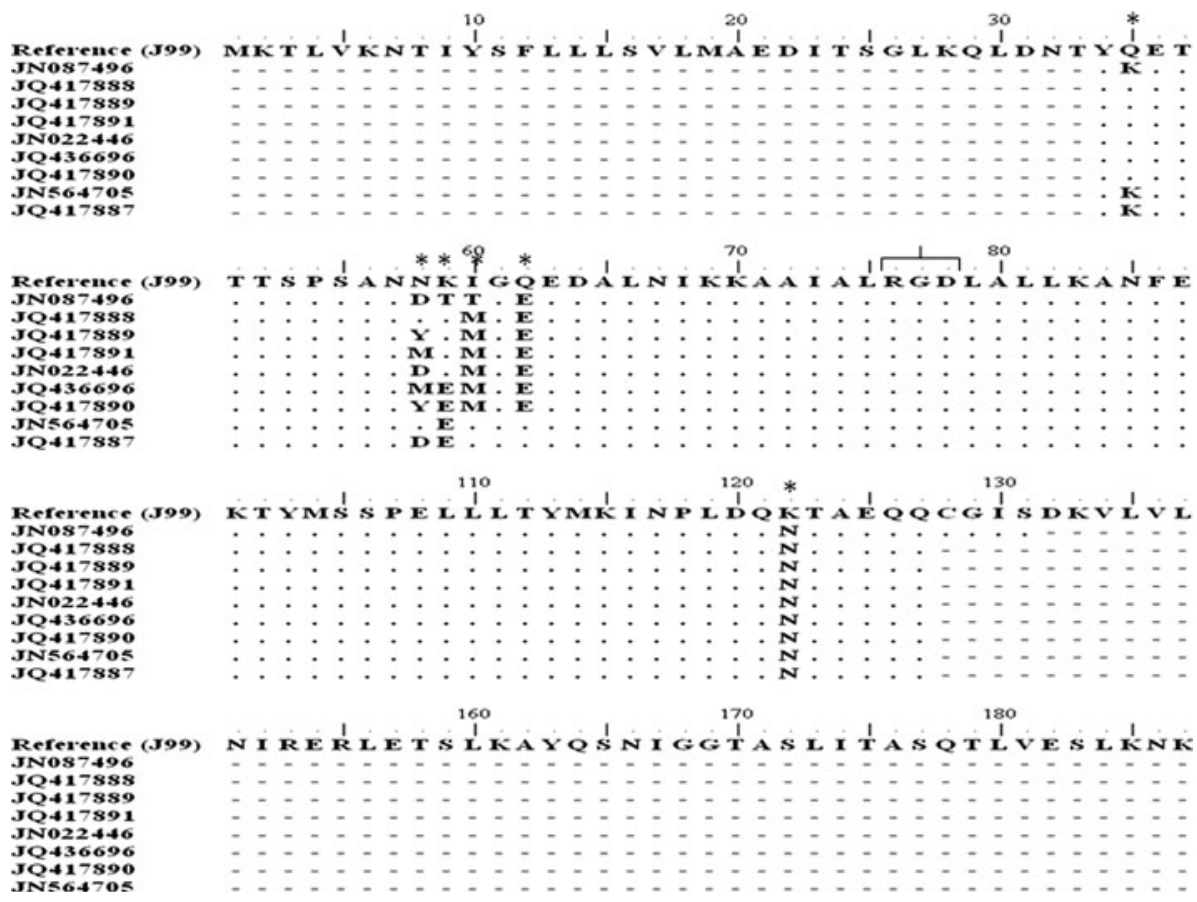

higher in patients with PUD than with GC (70 vs. $48 \%$, $P=0.045)$ and NUD (70 vs. $50 \%, P=0.038$ ).

\section{H. pylori genotyping}

In the present study, $86.6 \%(91 / 105)$ of the H. pylori isolates were positive for the $c a g L$ genotype by PCR. The prevalence of $c a g L$-positive $H$. pylori infection was uniformly distributed in patients with GC $(75 \% ; 18 / 24)$, NUD (86.6\%; 52/60), and PUD (100\%; 21/21). The cagA genotype was present in $86 \%(82 / 105)$ of our $H$. pylori isolates. Presence of $\operatorname{cagA}$ in GC, PUD, and NUD was $66.6 \%(16 / 24), 86 \%(18 / 21)$, and $88.3 \%$ (53/60), respectively. The majority of our $H$. pylori isolates, i.e., 78 (74.2\%), were positive for both cagA and cagL genotypes and were equally distributed in cases of GC $(12,50 \%)$, PUD $(18,86 \%)$, and NUD (48, $80 \%)$.

cagL amino acid sequence polymorphisms in disease population

The amplified products ( $354 \mathrm{bp}$ ) of the cagL gene from all clinical isolates were sequenced. After matching to the sequences available in the GenBank database, the nucleotide sequence of our isolates had uniform identities ( $>95 \%$ homology) to the cagL gene of the standard J99 strain, H. pylori strain 26695, and NCTC 11637. With the help of the expasy online translation tool, the amino acid sequences of $c a g L$ were translated for the selected isolates. Based on these predicted amino acid sequences, our all $H$. pylori isolates expressed the RGD motif (Fig. 1). There were no differences of the cagL amino acid sequence polymorphisms between patients with PUD and NUD $(P>0.05)$. However, the isolates from GC patients had significantly higher rates of D58 amino acid sequence polymorphism than those from PUD $(P=0.025)$ and NUD patients $(P=0.046)$. The presence of K59 was also found to be significantly higher in GC when compared to PUD $(P=0.011)$ and NUD $(P=0.010)($ Table 1$)$. Moreover, the presence of K59 usually accompanied D58 (Fig. 1), and these combined D58K59 polymorphisms had significantly higher rates of GC $[P=0.029$, odds ratio $(\mathrm{OR})(95 \%$ confidence interval, CI $=3.82(1.18-12.37)]$ (Table 2).

\section{Accession numbers}

Based on the combined 58 and 59 positions of $c a g L$ amino acid sequences, all cagL-positive isolates were divided into nine groups (DT, DK, NE, NK, YE, YK, MK, ME, and DE) and one representative sequence each from all groups was deposited in Gen-Bank with accession no. JN087496, JN022446, JN564705, JQ417888, JQ417890, JQ417889, JQ417891, JQ436696, and JQ417887, respectively (Supplementary Table SI).

\section{Discussion}

The prevalence of $H$. pylori in patients with GC in our study was low and had no association. Other tissue-based studies from India also failed to show an association between $H$. pylori infection and GC $[4,9,10]$. The chances 
Table 1 Differences in polymorphisms of $\mathrm{cag} L$ amino acid sequences of Helicobacter pylori isolates from patients with gastric cancer (GC), peptic ulcer disease (PUD), and nonulcer dyspepsia (NUD)

\begin{tabular}{llll}
\hline cagL amino acid polymorphisms & GC $(n=18)$ & PUD $(n=21)$ & NUD $(n=52)$ \\
\hline 35 & $13 / 5$ & $18 / 3$ & $40 / 12$ \\
Q/K & $72.2: 27.3$ & $85.7: 14.3$ & $80: 20$ \\
58 & $11 / 3 / 4 / 0$ & $5 / 11 / 3 / 2$ & $18 / 24 / 8 / 2$ \\
$\mathrm{D}^{\mathrm{a} / \mathrm{Y} / \mathrm{N} / \mathrm{M}}$ & $61.1: 16.6: 22.2: 0$ & $23.8: 52.3: 14.2: 9.5$ & $34.6: 46.1: 15.3: 3.8$ \\
$\mathrm{D} /$ others & $61.1: 38.9$ & $23.8: 62$ & $34.6: 57.7$ \\
59 & $4 / 12 / 2$ & $16 / 5 / 0$ & $37 / 15 / 0$ \\
$\mathrm{E} / \mathrm{K}^{\mathrm{b}} / \mathrm{T}$ & $22.2: 66.6: 11.1$ & $76.2: 23.8$ & $71.2: 28.8$ \\
$\mathrm{~K} / \mathrm{others}$ & $66.6: 33.4$ & $23.8: 76.1$ & $28.8: 71.2$ \\
60 & $11 / 7$ & $13 / 8$ & $30 / 22$ \\
$\mathrm{I} / \mathrm{M}$ & $61.1: 38.9$ & $62: 38$ & $57.6: 42.4$ \\
62 & $10 / 8$ & $18 / 3$ & $35 / 17$ \\
$\mathrm{Q} / \mathrm{E}$ & $55.6: 44.4$ & $85.7: 14.3$ & $67.3: 32.7$ \\
122 & $18 / 0$ & $21 / 0$ & $52 / 0$ \\
N/K & $100: 0$ & $100: 0$ & $100: 0$ \\
\hline
\end{tabular}

a D58GC versus D58NUD

(61.1 vs. $34.6 \%, P=0.046)$;

D58GC versus D58PUD (61.1

vs. $23.8 \%, P=0.025)$

b K59GC versus K59NUD

(66.6 vs. $28.8 \%, P=0.010$ );

K59GC versus K59PUD (66.6

vs. $23.8 \%, P=0.010$ )

Table 2 Association between amino acid polymorphism with gastric cancer (GC) and peptic ulcer disease (PUD)

\begin{tabular}{|c|c|c|c|c|c|c|}
\hline \multirow[t]{2}{*}{ Genotype $^{a}$} & \multicolumn{3}{|l|}{ GC } & \multicolumn{3}{|l|}{ PUD } \\
\hline & $P$ value & Odds ratio & $95 \% \mathrm{CI}$ & $P$ value & Odds ratio & $95 \% \mathrm{CI}$ \\
\hline DT & 0.063 & 0.889 & $0.755-1.047$ & - & - & - \\
\hline $\mathrm{DE}$ & 0.435 & 0.281 & $0.033-2.391$ & 1.00 & 0.796 & $0.193-3.281$ \\
\hline DK & 0.029 & $3.822^{\mathrm{b}}$ & $1.180-12.376$ & 0.494 & 0.503 & $0.099-2.553$ \\
\hline YE & - & - & - & 0.600 & 1.455 & $0.523-4.044$ \\
\hline YK & 0.145 & 0.320 & $0.082-1.246$ & 1.00 & 0.600 & $0.063-5.707$ \\
\hline YT & - & - & - & - & - & - \\
\hline $\mathrm{NE}$ & 0.103 & 5.00 & $0.763-32.765$ & 0.711 & 1.278 & $0.288-5.665$ \\
\hline NK & 1.00 & 0.961 & $0.094-9.869$ & - & - & - \\
\hline ME & - & - & - & - & - & - \\
\hline MK & - & - & - & 0.080 & 0.905 & $0.788-1.309$ \\
\hline MT & - & - & - & - & - & - \\
\hline
\end{tabular}

${ }^{a}$ Combined 58 and 59 position

b Positive association

of finding H. pylori in the biopsy specimens become less when the changes of chronic atrophic gastritis and intestinal metaplasia are set in the stomach. These changes lead to absence or decrease in the H. pylori load in the stomach, probably because of lack of nutrients for this organism [11]. So, these may be the probable reasons for the low prevalence of $H$. pylori infection when a tissue-based diagnosis is made.

No correlation of $\operatorname{cagA}$ could be established with GC and PUD, although $\operatorname{cag} A$ had been identified as a virulence marker and associated with increased severity of disease in some geographic regions [12]. The high prevalence of cagA (80-90\%) independent of disease status had been reported in other Indian studies as well [13, 14]. Therefore, we targeted the $c a g L$ genotype, which is responsible for the translocation of cagA into the gastric epithelium. The present study indicated $86.6 \%$ of our isolates were $c a g L$ positive and all carried the RGD motif in their amino acid sequences. Studies from Malaysia, Singapore, and Taiwan reported $>85 \%$ prevalence of $c a g L$ in their $H$. pylori isolates, and the results are concordant with the current finding $[15,16]$. Moreover, the presence of RGD motif in all the current isolates suggests that the CagA translocation is mediated through an RGD-dependent pathway. Therefore, it is rational to check whether the cagL amino acid sequence polymorphisms correlate with clinical disease. Previously, a study from Taiwan reported a higher rate of the amino acid sequence polymorphisms Y58 and E59 in GC patients $(P<0.05)$ and concluded that cagL-Y58E59 isolates possibly exert stronger acid suppression during chronic infection [16]. On the other hand, the results of Saha et al. [17] help in part to explain that CagL may 
dissociate ADAM17 of integrin a5b1 to contribute to hypochlorhydria during $H$. pylori infection. In the present study, different amino acid polymorphisms, D58 and K59, were significantly associated with GC. However, an experimental approach that probes mechanistically the observed associations between D58K59 cagL amino acid polymorphisms and $\mathrm{GC}$ is required to reach any conclusion.

\section{Conclusion}

In summary, the majority of Indian $H$. pylori isolates were cagL positive and all such strains carried the RGD motif. Patients infected with cagL-genopositive $H$. pylori strains having cagL-D58K59 amino acid polymorphisms had a 3.8-fold increased risk for development of gastric cancer. However, further studies are required to know the exact role of particular cagL amino acid polymorphisms in the pathogenicity of $H$. pylori-associated gastroduodenal diseases.

Acknowledgments This work was supported by the research fund of Sanjay Gandhi Postgraduate Institute of Medical Sciences, Lucknow, India (PGI/IMP/EC/48/2009). Sanket Kumar Shukla and Aparna Tripathi gratefully acknowledge the Department of Biotechnology, Govt. of India (DBT) and Indian Council of Medical Research, New Delhi, for a senior research fellowship through Grant No. (DBT-JRF)/09-10/634 and 80/625/2009-ECD-I, respectively.

\section{References}

1. Kate V, Ananthakrishnan N. Helicobacter pylori and gastric carcinoma: evidence for the link. Natl Med J India. 2000;13:329.

2. Atherton JC. The pathogenesis of Helicobacter pylori-induced gastroduodenal diseases. Annu Rev Pathol. 2006;1:63-96.

3. Covacci A, Rappuoli R. Tyrosine-phosphorylated bacterial proteins: Trojan horses for the host cell. J Exp Med. 2000; 191:587-92.

4. Saxena A, Shukla S, Prasad KN, Ghoshal UC. Virulence attributes of Helicobacter pylori isolates and their association with gastroduodenal disease. Indian J Med Res. 2011;133:514-20.

5. Kwok T, Zabler D, Urman S, Rohde M, Hartig R, Wessler S, Misselwitz R, Berger J, Sewald N, Konig W, Backert S.
Helicobacter exploits integrin for type IV secretion and kinase activation. Nature (Lond). 2007;449:862-6.

6. Singh M, Prasad KN, Yachha SK, Krishnani N. Genotypes of Helicobacter pylori in children with upper abdominal pain. J Gastroenterol Hepatol. 2003;18:1018-23.

7. Covacci A, Censini S, Bugnoli M, Petracca R, Burroni D, Macchia G, Massone A, Papini E, Xiang Z, Figura N, et al. Molecular characterization of the $128-\mathrm{kDa}$ immunodominant antigen of Helicobacter pylori associated with cytotoxicity and duodenal ulcer. Proc Natl Acad Sci USA. 1993;90:5791-5.

8. Occhialini A, Marais A, Urdaci M, Sierra R, Munoz N, Covacci A, Megraud F. Composition and gene expression of the cag pathogenicity island in Helicobacter pylori strains isolated from gastric carcinoma and gastritis patients in Costa Rica. Infect Immun. 2001;69:1902-8.

9. Khanna AK, Seth P, Nath G, Dixit VK, Kumar M. Correlation of Helicobacter pylori and gastric carcinoma. J Postgrad Med. 2002;48:27-8.

10. Sivaprakash R, Rao UA, Thyagarajan SP, Ramathilakam B, Jayanthi V. Investigation for the prevalence of Helicobacter pylori infection in patients with gastric carcinoma in Madras, India. Jpn J Med Sci Biol. 1996;49:49-56.

11. Konturek PC, Kania J, Konturek JW, Nikiforuk A, Konturek SJ, Hahn EG. H. pylori infection, atrophic gastritis, cytokines, gastrin, COX-2, PPAR gamma and impaired apoptosis in gastric carcinogenesis. Med Sci Monit 2003;9:SR53-66.

12. Blaser MJ, Perez-Perez GI, Kleanthous H, Cover TL, Peek RM, Chyou PH, Stemmermann GN, Nomura A. Infection with Helicobacter pylori strains possessing cagA is associated with an increased risk of developing adenocarcinoma of the stomach. Cancer Res. 1995;55:2111-5.

13. Mishra KK, Srivastava S, Dwivedi PP, Prasad KN, Ayyagari A. UreC PCR-based diagnosis of Helicobacter pylori infection and detection of $c a g A$ gene in gastric biopsies. Indian $\mathrm{J}$ Pathol Microbiol. 2002;45:31-7.

14. Mukhopadhyay AK, Kersulyte D, Jeong JY, Datta S, Ito Y, Chowdhury A, Chowdhury S, Santra A, Bhattacharya SK, Azuma T, Nair GB, Berg DE. Distinctiveness of genotypes of Helicobacter pylori in Calcutta, India. J Bacteriol. 2000;182:3219-27.

15. Schmidt HM, Andres S, Nilsson C, Kovach Z, Kaakoush NO, Engstrand L, Goh KL, Fock KM, Forman D, Mitchell H. The cag PAI is intact and functional but HP0521 varies significantly in Helicobacter pylori isolates from Malaysia and Singapore. Eur J Clin Microbiol Infect Dis. 2010;29:439-51.

16. Yeh YC, Chang WL, Yang HB, Cheng HC, Wu JJ, Sheu BS. H. pylori cagL amino acid sequence polymorphism Y58E59 induces a corpus shift of gastric integrin alpha5beta1 related with gastric carcinogenesis. Mol Carcinog. 2011;50:751-9.

17. Saha A, Backert S, Hammond CE, Gooz M, Smolka AJ. Helicobacter pylori CagL activates ADAM17 to induce repression of the gastric H, K-ATPase alpha subunit. Gastroenterology. 2011;139:239-48. 